\title{
Los cirujanos y los medios de comunicación, relaciones peligrosas
}

\author{
Álvaro SANABria
}

Palabras clave: cirugía general; periodismo médico; docentes médicos; rol del médico.

El cirujano, como la medicina en general, está inmerso en un mundo de comunicaciones, y la comunicación por los diferentes medios, impresos, hablados, televisivos y digitales, es una realidad de la vida diaria. Por otra parte, el cirujano debe comunicar a su paciente y a su familia todo lo pertinente a la intervención quirúrgica que se propone, o describir la que acaba de realizar. También se debe a la opinión pública, a fin de difundir los alcances de la cirugía moderna o cuando se realiza un procedimiento complejo por primera vez o se emplea una nueva técnica.

No obstante, en Colombia, como en muchos países de Latinoamérica, no existe el verdadero periodismo científico, como sí sucede en las naciones del primer mundo. Basta ver el Science Times del New York Times que aparece como la sección especial los días martes, para comprender qué es periodismo científico: rigor, precisión y exactitud absolutos en la narración, hasta el punto de que muchos coleccionan el Science Times al igual que una revista científica.

Cuando se transmite la descripción de un acto médico, y especialmente de un acto quirúrgico, a un periodista

Profesor, Departamento de Cirugía, Universidad de Antioquia; cirujano de Cabeza y Cuello, Fundación Colombiana de Cáncer, Clínica Vida, Medellín, Colombia

Fecha de recibido: 27 de marzo de 2017

Fecha de aprobación: 19 de abril de 2017

Citar como: Sanabria Á. Los cirujanos y los medios de comunicación, relaciones peligrosas. Rev Colomb Cir. 2017;32:171-5. que no tiene formación en periodismo científico y que no está versado en la materia, necesariamente se corre el riesgo de que la noticia aparezca exagerada o francamente distorsionada.

Debido a una reciente experiencia personal, se escriben estas líneas con el fin de advertirle al cirujano lo que le puede ocurrir con el relato de un procedimiento quirúrgico, y de aconsejarle que cuando se le haga una entrevista le exija al periodista que le muestre el escrito para que el cirujano pueda hacerle las correcciones pertinentes. Esto en ninguna forma atenta contra la libertad de prensa, sino que lleva a que este tipo de noticias estén ceñidas a la realidad.

Habiendo sido citado en calidad de cirujano de cabeza y cuello de una institución de la ciudad a participar en una rueda de prensa en la que se discutiría el caso exitoso de un paciente en cuyo tratamiento había participado, la noticia fue presentada en forma distorsionada y causó gran impacto en la opinión pública. El caso era simple: un paciente joven con un tumor de lengua y sin antecedentes de riesgo, a quien se le propuso y se le practicó un procedimiento complejo que involucraba una resección mayor de la lengua y la consecuente reconstrucción con un colgajo microquirúrgico. El propósito de la entrevista era explicar a los medios el procedimiento practicado y aclarar las dudas que pudieran surgir. Así se hizo, sin otra pretensión que la de hacer claridad sobre lo realizado.

El procedimiento es uno más de los efectuados en muchos lugares del mundo y en el país, sin que fuera una novedad o una innovación. Sin embargo, unas horas después, se generó un vórtice de inconsistencias e 
imprecisiones en la radio, la televisión y la prensa ${ }^{1,2}$. De la noche a la mañana, el nombre construido durante 20 años de vida profesional -respaldado por títulos académicos en universidades serias del país y del extranjero- se vio amenazado por una enorme cantidad de enunciados, titulares, extras y demás fenómenos informativos inmediatistas, al tiempo que se recibieron felicitaciones y recriminaciones por algo que nunca se dijo, que nunca se hizo y que jamás se afirmó, pero que de un plumazo se convirtió en la verdad absoluta y efímera del día. Tales hechos me obligaron a no responderles a los medios y a dejar de emitir opiniones en el mismísimo seno de la comunidad académica y social. Obviamente, esta situación no es nueva y existen ejemplos previos en la literatura ${ }^{3}$.

Las reacciones a las noticias son un tema importante de abordar. La aparición de un colega en los medios con una nota controversial jamás dejará satisfechos a todos los espectadores. Los que conocen de primera mano el caso pueden llegar a entender que lo expuesto no corresponde exactamente a lo que aparece en un periódico o en una nota informativa de televisión o de radio. Pero aquellos lejanos del tema, e incluso los mismos protagonistas del episodio, pueden interpretar la controversia como una consecuencia del ánimo mercantilista del galeno, la inminente existencia de una conspiración para cambiar el orden mundial o, simplemente, la confirmación de la maldad que hay dentro de cada ser humano. Hoy en día, con el auge de las redes sociales, estas posiciones extremas ayudan a avivar el fuego de la desinformación. Vienen felicitaciones unas, y acusaciones otras, injustas todas, que se hacen con el objetivo de 'poner en su lugar' al médico, como si él fuera el responsable de que el mensaje se haya interpretado erróneamente. Tampoco quedarán muy contentos los protagonistas del caso. El cirujano probablemente acusará al periodista de tergiversar las opiniones y así lo hará saber por los medios a la mano y tomará una posición defensiva ante cualquier futura comunicación con la prensa. El periodista, a su vez, acusará al cirujano de no ser claro y de manipular la información para obtener un beneficio personal, y se comportará igual cuando tenga que encontrarse de nuevo con otro médico.

La vida moderna está llena declaraciones efímeras. Y son los medios la máquina que hace esto posible, especialmente la televisión y la radio que, en cápsulas de pocos segundos, pretenden resumir conocimientos que toman años en asentarse. En los periódicos, al menos lo que se escribe, se queda para siempre y es posible citarlo y criticarlo. Pero en estos medios etéreos, en los que poco queda guardado y la interpretación es un elemento que no se contempla, lo que se dice puede ser tergiversado, acomodado, recortado y hasta renovado, por obra y magia de la tecnología. Sin embargo, la vida gira alrededor de los medios, tres veces al día frente a una pantalla de televisión, unas cuantas horas mientras se va al trabajo escuchando la radio, o unos minutos mientras se toma un café y se ojea el periódico. Finalmente, uno se convierte en partícipe de esa marea informativa y no es posible alejarse de ella. La vida del médico depende de la información. Las novedades, los descubrimientos, los debates, etc. llegan a través de los medios y el médico los usa para transmitir un mensaje o defender un interés. Los cambios en la práctica profesional ocurren como consecuencia del esfuerzo de los médicos por demostrar los hechos y de los periodistas por convertirlos en un mensaje asequible para el público lego ${ }^{4}$.

Entonces, ¿cómo debe ser la relación entre cirujanos y medios para que el interés de la trasmisión de un mensaje que puede cambiar la vida de las personas llegue en la forma más diáfana y precisa posible? ¿Cómo se puede interactuar con los periodistas sin que lo que se pretende decir se desfigure? ¿Cómo se puede mantener la transparencia del mensaje en medio de los intereses de las partes?

La respuesta no es fácil y es improbable que en estos pocos párrafos logre siquiera arañar un poco de verdad. Pero el hacer el ejercicio contribuye, no solo a la autorreflexión personal que impide que se repitan los errores del pasado, sino a avivar la discusión alrededor del tema y a educar, o, al menos, prevenir a aquellos que pueden verse enfrentados a tal situación.

Terry Schraeder, médica y periodista, dice que el conflicto existente entre médicos y periodistas, más que un problema de intereses y malicia, es de ignorancia, la cual lleva a la mala comunicación, al desentendimiento y a la confusión del mensaje que se transmite ${ }^{5}$. Dice Schraeder:

“[...] Los periodistas se relacionan con los médicos pensando que protegen al público de un enemigo, mientras que los médicos se quejan de la poca educación y preocupación por entender y reportar los 
hechos de forma precisa, de la manipulación externa y del sensacionalismo [...]".

El fin último del periodismo científico es mostrar la verdad, pero hay que reconocer la realidad de que ambos, cirujanos y periodistas, son humanos y que la ciencia en sí es una actividad humana que no está libre de sesgos y que los intereses de cada una pueden matizar esta verdad ${ }^{6,7}$.

El primer paso en la consecución de una relación confiable y duradera es entender las dificultades del otro. Los periodistas deben entender que el conocimiento médico es muy amplio y de rápido recambio, que la toma de decisiones depende de múltiples factores e interacciones y que se hace en medio de la incertidumbre, y que siempre hay un equilibrio entre el costo y el beneficio que no es extrapolable a todas las personas y a todas las enfermedades. Un sano escepticismo ante las versiones de un médico es una buena vacuna para los errores, y buscar la verificación de otras fuentes siempre es loable, sobre todo si se tiene en cuenta que una noticia equivocada puede costar vidas y carreras ${ }^{8}$. Pero, también, los médicos deben comprender que los periodistas tienen restricciones de tiempo, espacio y conocimiento (entendiendo que ellos no son médicos), que en un día de trabajo tienen que abordar tópicos de salud y de medicina diametralmente opuestos, que deben mantener un equilibrio entre la confiabilidad y el interés particular de sus fuentes, y que todos los días las verdades cambian, producto de la investigación y el desarrollo científico.

De esta experiencia se desprenden algunas enseñanzas que conviene compartir, a fin de mostrar cómo es de fácil sumergirse en estos laberintos y cómo evitar cometer el mismo error en el que inocentemente se puede caer, pero en el cual, inocentemente también, se condena ${ }^{9-12}$.

No asumir que el caso es simple. Para quienes viven en el mundo complejo de la enfermedad y en el más intricado de la cirugía, procedimientos como un trasplante hepático son tareas cotidianas, relativamente simples y fácilmente asequibles con entrenamiento y dedicación. Pero eso no es cierto para las personas que no hacen parte del círculo de los actores del sistema de salud. Para los demás mortales, estos actos se hallan en un terreno intermedio entra la magia y el milagro, de tal forma que si a algún periodista le llama la atención lo que el cirujano hace, es necesario percatarse de que eso que para los cirujanos es simple, para los demás es algo que solo compete a unos pocos 'iluminados'. Con esa diferencia de percepciones, la simplicidad desaparece porque los legos quieren saber al detalle y en un instante lo que el cirujano demoró años en conocer, planificar y realizar. No es posible encontrar el equilibrio a corto plazo y siempre se exaltarán nimiedades de las que nunca se pensó que ni siquiera fueran relevantes. Así que se debe tomar un tiempo para explicar antes de dar declaraciones oficiales y, también, asegurarse de que quien lo escucha entiende la complejidad del problema que se discute.

No asumir que hay tiempo para explicar después. Los cirujanos están acostumbrados a discutir los pacientes en jornadas, a veces interminables, llenas de debates y objeciones, alternativas y callejones sin salida, en las cuales, al final, es una combinación especial entre el conocimiento, la intuición y el arrojo lo que permiten tomar una decisión. Esto no es así para los medios de comunicación. Por el contrario, ellos quieren una verdad absoluta, nítida, clara y libre de incertidumbres, lo cual no existe en medicina ${ }^{13}$. Se puede explayar tratando de explicar una situación y esta va a terminar resumida en una frase lacónica que dura unos segundos. Tal síntesis necesariamente implica pérdida de información. Así, lo que se dijo puede ser reducido a unas pocas palabras que pueden dejar una impresión completamente contraria a lo que se intentó transmitir. Es por ello que se debe asegurar ver el producto antes de ser publicado, porque, una vez publicado, no hay vuelta atrás.

No asumir que las cosas se presentarán en su justa medida. En la jerarquía de los procedimientos existen unos menos y otros más complicados, dependiendo del esfuerzo que se debe invertir y de la tecnología usada. Es claro para todos que una hernia inguinal es mucho menos compleja que una cirugía de corazón, así la primera se haga con los instrumentos y avances más modernos, para poner un ejemplo, usando un robot. Pero esa diferencia no es clara para los demás. En el imaginario de las personas ajenas a la medicina, y especialmente a la cirugía, tecnología implica complejidad y deshumanización, y los cirujanos han contribuido a acentuar dicho engendro, de tal forma que un procedimiento complejo que implica un esfuerzo de disección y manejo delicado de los tejidos, como puede ser una disección vascular, queda desplazado por uno menos complejo pero que 
llama la atención por lo extraño de su abordaje, por las ventajas potenciales o por unos resultados con beneficios marginales, como puede ser el ejemplo de una cirugía NOTES (Natural Orifice Transluminal Endoscopic Surgery). Entonces, la transmisión del mensaje es influenciada por esta divergencia sensorial y lo que es simple se torna más complejo de lo que realmente es por obra y magia de los medios. Es por ello que se debe clarificar el grado de complejidad de las intervenciones sobre las que se va a dialogar y a hacer comparaciones, a fin de entender la magnitud de lo que se ejecuta.

No asumir que el contexto se tendrá en cuenta. Si un día alguien en Ciudad del Cabo a finales de la década de los sesenta decidió hacer un trasplante de corazón, esto aparece como un hecho fantástico, casi divino. Pensar que en un país del tercer mundo, con pocos recursos, pueda realizarse una proeza como esa mueve a los medios ávidos de 'chivas' y noticias. Pero, si el mismo procedimiento se hace en un paciente bastante más complejo y en un hospital dotado con la mejor tecnología y lo que se pretende es mostrar que después de 100 casos consecutivos no hay mortalidad, esto carece de interés periodístico, pues es uno más en el infinito mundo de las cosas comunes que se practican en estos hospitales. Se sabe que la realidad no es esa. El contexto determina los logros. Cuando se tiene acceso a los recursos, hacer proezas es mucho más fácil que cuando se carece de ellos.

No obstante, en la actualidad eso no es tan importante: lo que vale es el flechazo informativo, la rareza, el hecho increíble, la persecución del 'primer caso'. Es así como los medios soslayan el contexto y ensalzan aquello que tenga posibilidades de llamar la atención, en desmedro de otros aspectos que sí son fundamentales. Se debe ser muy explícito sobre qué de extraordinario tiene su experiencia y, si considera que no lo es, enunciarlo y decir qué es lo que impactará a quien vea la noticia.

No asumir que el resultado final es importante. Para el cirujano, el éxito de un procedimiento quirúrgico se mide por la suma de éxitos parciales que llevan a la supervivencia y mejoría de la calidad de vida del paciente. Si después de una gastrectomía el paciente no se complica y logra vivir una vida parecida a la normal, esto es un éxito, pero para los medios ello no es así.
Son los logros pequeños, mínimos, pero llamativos y sorprendentes, los que definen el éxito. Lo que pase al final ya no será una noticia.

Siguiendo con el ejemplo del primer trasplante cardiaco, todos conocen el nombre del cirujano que lo realizó, unos pocos el nombre del paciente y la minoría saben que Louis Washkansky murió 18 días después, sin haber recibido un beneficio personal mayor.

Entonces, la meta para el cirujano y para el periodista es diferente. Mientas el cirujano piensa en el resultado final, el periodista se concentra en la etapa, y esto lleva a perder la perspectiva. Es mucho más atractivo extraer la glándula tiroides por la boca que contar que, al final, el paciente terminó con una traqueostomía por una lesión bilateral de nervios. Lo que impacta y sacude es ese hecho inicial que nada tiene que ver con el resultado final. Por ello, se debe hacer énfasis en el resultado final, en la calidad de vida, en la posibilidad de que el paciente pueda disfrutar de su familia, que pueda trabajar, más que en el logro técnico de la intervención. El árbol no puede hacer perder de vista el bosque.

Finalmente, no asumir que se pueden hacer correcciones. Los medios siempre tienen la razón. Si se es víctima de todo lo que se explica en los párrafos anteriores y se decide aclarar la situación, caerá en picada, porque esto funciona como un terreno movedizo. Entre más se intenta salir, más se hunde, por la simple razón de que la noticia mueve las vísceras de las personas, mientras el cirujano apunta al intelecto. Es por ello que resulta mejor no intentarlo, y más vale recordar que las noticias solo duran unas cuantas horas de hoy y que mañana habrá otra mucho más interesante que sepultará la suya.

Como consejo final, se debe advertir sobre la importancia de ser cauto cuando se participa en actividades que incluyan entrevistas. Si es absolutamente necesaria, se debe seguir el buen consejo de abogado de responder explícitamente lo que se le pregunta, idealmente con unos cuantos monosílabos. Si se puede dejar una nota por escrito, mucho mejor, las palabras escritas no se las lleva el viento. Y no debe dejarse seducir por esa vanidad propia de los seres humanos de querer ser reconocidos, para no pasar del anonimato al desprestigio. 


\section{Referencias}

1. La increíble historia del hombre con el primer implante de lengua en Medellín, 2017. Fecha de consulta: 20 de marzo de 2017. Disponible en: http://www.bluradio.com/medellin/ la-increible-historia-del-hombre-con-el-primer-trasplante-delengua-en-medellin-134704.

2. Primera reconstrucción de una lengua completa en Antioquia. 2017. Fecha de consulta: 20 de marzo de 2017. Disponible en: http://www.elcolombiano.com/colombia/salud/primera-reconstruccion-de-una-lengua-completa-en-antioquia-XA6165624.

3. Coombes R. BMJ in 'smug docs' storm. BMJ. 2008;336:1340-1.

4. Rubin R. Navigating the minefields of medicine and journalism. JAMA. 2015;314:545-7.

5. Schraeder T. A chasm of distrust in medical reporting. 2003 Fecha de consulta: 20 de marzo de 2017. Disponible en: http:// niemanreports.org/articles/a-chasm-of-distrust-in-medicalreporting/.

6. Borel B. The problem with science journalism: We've forgotten that reality matters most. Media. 2015. Fecha de consulta: 20 de marzo de 2017. Disponible en: https://www.theguardian. com/media/2015/dec/30/problem-with-science-journalism2015-reality-kevin-folta.

7. Bosman J. Reporters find science journals harder to trust, but not easy to verify. N Y Times Web. 2006:C1, C3.

8. Schulz K. Why journalists get the big things wrong. Timeframes. 2010. Fecha de consulta: 20 de marzo de 2017. Dis- ponible en: http://content.time.com/time/specials/packages/ article/0,28804,2032304_2032746_2033037,00.html.

9. Entwistle V. Reporting research in medical journals and newspapers. BMJ. 1995;310:920-3.

10. Lewis S. How to beat the media in the climate street fight. World View. 2010. Fecha de consulta: 20 de marzo de 2017. Disponible en: http://www.nature.com/news/2010/101103/full/468007a. html.

11. Editors PLM, Barbour V, Clark J, Peiper L, Veitch E, Wong M, et al. False hopes, unwarranted fears: The trouble with medical news stories. PLoS Med. 2008;5:e118.

12. Vallano-Ferraz A, Llop-Rius R, Bosch-Ferrer M, Danes-Carreras I. Drug notices in the health supplement of Spanish newspapers. Med Clin (Barc). 2005;124:754-5.

13. Making sense of uncertainty. 2013. Fecha de consulta: 25 de marzo de 2017. Disponible en: http://senseaboutscience.org/ wp-content/uploads/2016/11/Makingsenseofuncertainty.pdf.

Correspondencia: Álvaro Sanabria, MD

Correo electrónico: alvarosanabria@gmail.com Medellín 